\title{
Feasibility of rapid quantitation of stratum corneum lipid content by Fourier transform infrared spectrometry
}

\author{
Jui-Chen Tsai ${ }^{\mathrm{a}, *}$, Yu-Li Lo ${ }^{\mathrm{b}}$, Ching-Yu Lin ${ }^{\mathrm{a}, * *}$, Hamm-Ming Sheu ${ }^{\mathrm{c}}$ and Jui-Che Lin ${ }^{\mathrm{d}}$ \\ ${ }^{a}$ Institute of Clinical Pharmacy, College of Medicine, National Cheng Kung University, Tainan 701, \\ Taiwan \\ ${ }^{\mathrm{b}}$ Department of Pharmacy, Chia-Nan University of Pharmacy and Science, Tainan Hsien 717, Taiwan \\ ${ }^{\mathrm{c}}$ Department of Dermatology, College of Medicine, National Cheng Kung University, 1 University Rd., \\ Tainan 701, Taiwan \\ ${ }^{\mathrm{d}}$ Department of Chemical Engineering, College of Engineering, National Cheng Kung University, \\ 1 University Rd., Tainan 701, Taiwan
}

\begin{abstract}
The permeability barrier of skin resides in the stratum corneum, and its properties are mediated by a series of lipid multilayers, enriched in ceramides, cholesterol, and free fatty acids, segregated within the stratum corneum (SC) interstices. SC lipid content is usually determined by gravimetric methods in conjunction with high performance thin layer chromatography, but these methods are time-consuming and involve hazardous solvents. The objective of the present study was to develop a method of measuring SC lipid content by Fourier transform infrared spectrometry (FTIR) that is fast and requires no solvents. The IR spectra of isolated porcine SC sheets were recorded using a FTIR spectrometer. SC lipid content was determined by gravimetric methods using chloroform-methanol extraction. The peak area of both the $\mathrm{CH}_{2}$ symmetric $\left(2850 \mathrm{~cm}^{-1}\right)$ and asymmetric $\left(2920 \mathrm{~cm}^{-1}\right)$ stretching bands in the IR spectra of progressively solvent-extracted porcine SC sheets decreased with increasing amount of SC lipids removed. When spectral analysis was performed by curve-fitting using GRAMS/32 software between 3000 to $2800 \mathrm{~cm}^{-1}$, peak area ratios of $\mathrm{CH}_{2}$ to $\mathrm{CH}_{3}$ asymmetric stretching bands in the IR spectra of 46 isolated porcine SC samples were correlated to SC lipid content $\left(R^{2}=0.90\right)$, with the standard error of measurement of $1.91 \%$. The study demonstrated the feasibility of using FTIR technique to rapidly and accurately measure SC lipid content.
\end{abstract}

Keywords: Stratum corneum, infrared spectrometry, lipid content, quantitation, deconvolution

\section{Introduction}

Stratum corneum (SC), the outermost layer of the skin, serves as a barrier to both water loss and to penetration of exogenous substances [1,2]. Such barrier properties are mediated by a series of lipid multilayers, enriched in ceramides, cholesterol and free fatty acids, segregated within the SC interstices [3]. Various studies have indicated that SC lipid content is defective in certain physiologic and pathologic states that are accompanied by compromised barrier function, such as due to essential fatty acid deficiency, psoriasis, ichthyoses, atopic dermatitis, UV irradiation, topical corticosteroid treatment and for the premature newborn, etc. [4-9]. SC lipid content of these physiologic and pathologic states is usually

\footnotetext{
${ }^{*}$ Corresponding author: Tel.: +886 6 2353535, ext. 5687; Fax: +886 6 2373149; E-mail: jctsai@mail.ncku.edu.tw.

${ }^{* * *}$ Present address: Pfizer Limited, Tamsui, Taiwan.
} 
determined by gravimetric measurement and high performance thin layer chromatography following organic solvent extractions $[8,10]$. These procedures are time-consuming, are rather tedious to carry out, and involve hazardous solvents.

Fourier transform infrared (FTIR) spectroscopy, which is used to measure the vibrational modes of the functional groups of molecules, is sensitive to molecular structure, conformation and environment of the biomolecules [11]. It has been widely utilized to investigate qualitatively and semi-quantitatively the effect of various treatments, such as heat, vehicles and penetration enhancers, on the SC lipid morphology [12-15]. The area under the $\mathrm{C}-\mathrm{H}$ stretching absorbance in the spectral range between 3000 and $2800 \mathrm{~cm}^{-1}$ have been used to report on the relative amount of lipid present as a function of depth into the SC in vivo [16]. Moreover, in clinical laboratory, the method has also been applied to quantitate fecal fat [17], and to analyze plasma levels of cholesterol and triglycerides [18].

Here we describe a method for rapidly and accurately measuring lipid content of isolated SC sheets, which requires no reagents and needs no sample preparation. It is performed on a FTIR spectrometer equipped with a simple sample holder for transmission IR measurements, and a statistical package is employed to deconvolute the IR spectra.

\section{Materials and methods}

\subsection{Isolation of porcine $S C$}

Porcine skin was obtained from a local abattoir and stored at $-70^{\circ} \mathrm{C}$. Sheets of $\mathrm{SC}$ were prepared by treating porcine skin, dermatomed to $500 \mu \mathrm{m}$, with $0.5 \%$ trypsin, in phosphate-buffered saline, $\mathrm{pH} 7.4$, for $18 \mathrm{~h}$ at $4{ }^{\circ} \mathrm{C}$. At the end of incubation period, the $\mathrm{SC}$ was separated from the dermis. Any remaining epidermal cells were then removed by mild agitation of the SC sheets [19]. Samples were dried overnight on a Speed-Vac concentrator and stored in a desiccator.

\subsection{FTIR spectroscopy}

FTIR spectra of isolated porcine SC were recorded using a Nicolet Magna 560 FTIR spectrometer (Madison, WI, USA) equipped with a Whatman Model 75-45 FTIR purge gas generator (Haverhill, MA, USA), a liquid nitrogen-cooled mercury-cadmium-telluride detector, and OMNIC E.S.P. software for data acquisition and spectral analysis. In this study, each spectrum obtained was the average of 64 scans. Resolution was set at $4 \mathrm{~cm}^{-1}$. Under these conditions, it required 1-2 min for acquisition of a spectrum over the wavelength range of $4000-650 \mathrm{~cm}^{-1}$. Measurements were taken at ambient laboratory conditions with $20-25^{\circ} \mathrm{C}$ and $50-70 \%$ relative humidity. SC sheets obtained from different body sites of the pig were trimmed to squares of $1.2 \times 1.2 \mathrm{~cm}^{2}$. Each sheet was placed between two $\mathrm{CaF}_{2}$ windows of sample holder in different orientations, such that one spectrum was collected near each corner of the sheet and a total of 4 spectra were collected for each SC sheet.

\subsection{Experiments}

Two sets of experiments were performed. After brief rinsing with a small amount of cold hexane, a single piece of porcine SC sheet was progressively extracted with $20 \mathrm{ml}$ chloroform/methanol mixtures of different volume ratios (from $1: 20$ to $2: 1$ ) for various time periods (from $10 \mathrm{~min}$ to overnight). IR spectrum of the same SC sheet was collected prior to and after each solvent extraction, according to 
the procedures described above. Lipid weight in each solvent extract was determined by the gravimetric method described as follows. In a separate set of experiments, isolated porcine SC sheets obtained from different body areas were briefly rinsed with a small amount of cold hexane. IR spectra of each sheet were recorded, and its lipid content was again determined gravimetrically.

\subsection{Gravimetric measurement of SC lipids}

The lipid extractions of the ground porcine SC were done at $2 \mathrm{~h}$ intervals with each of a series of chloroform/methanol mixture $(2: 1,1: 1$, and $1: 2$ by volume) [10]. The lipid extracts were filtered through a solvent-resistant $0.5 \mu \mathrm{m}$ PTFE Millipore filter to remove nonlipid debris, concentrated under nitrogen, and dried in a vacuum. The residue was then dissolved in $2 \mathrm{ml}$ of chloroform/methanol $(2: 1)$ mixture. Total SC lipid weight was determined by weighing of the lipid residue from evaporating a $200 \mu \mathrm{l}$ aliquot on a aluminum pan on a Mettler balance (Model AT20, sensitivity of $2 \mu \mathrm{g}$ ).

\subsection{Spectral analysis}

Spectral manipulations were performed with baseline correction function using OMNIC (Fig. 1) and with curve-fitting function using GRAM/32 (Galactic Industries, Salem, NH, USA) (Fig. 2). The peak

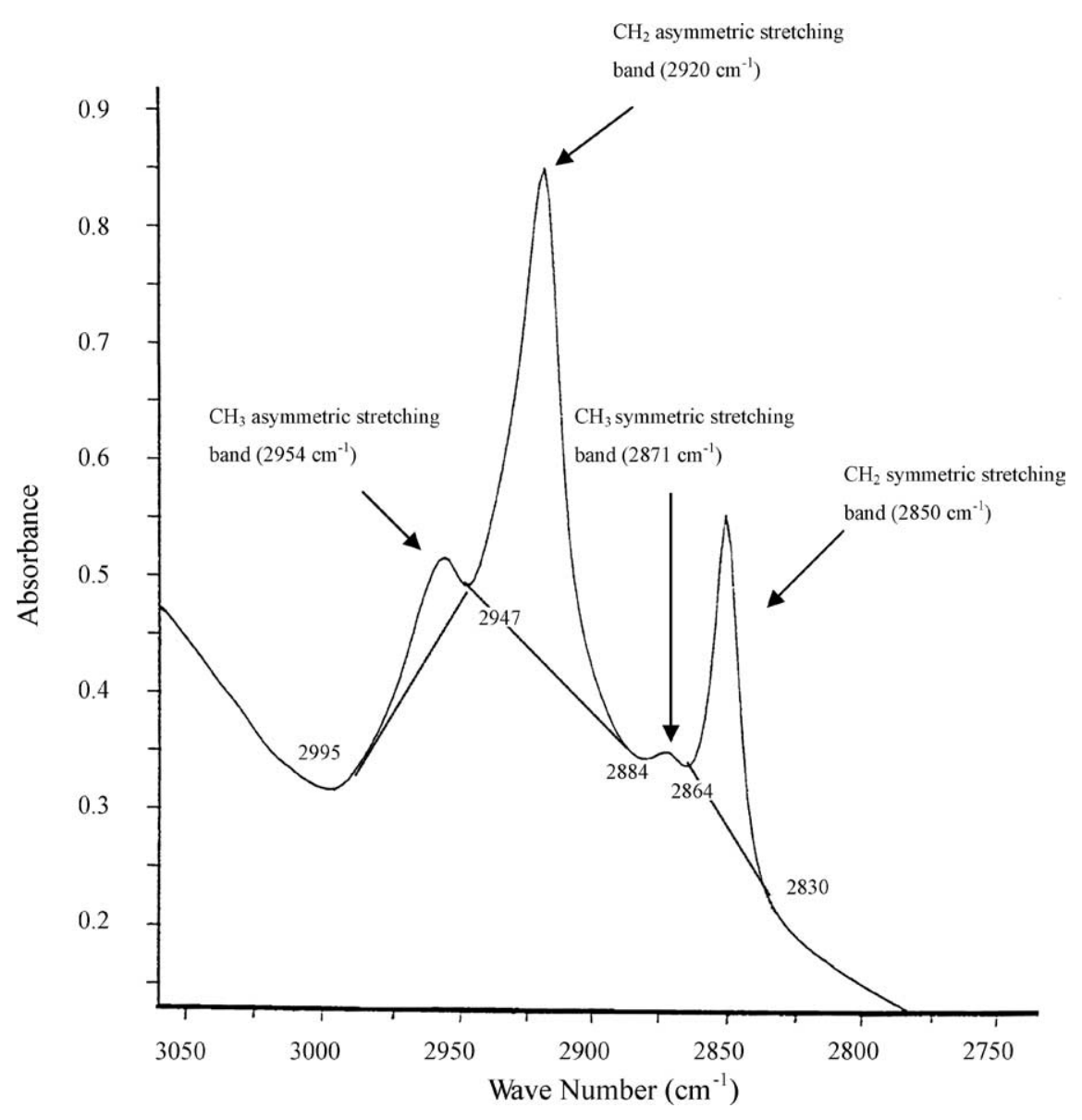

Fig. 1. Spectral analysis with baseline correction using OMNIC software. 


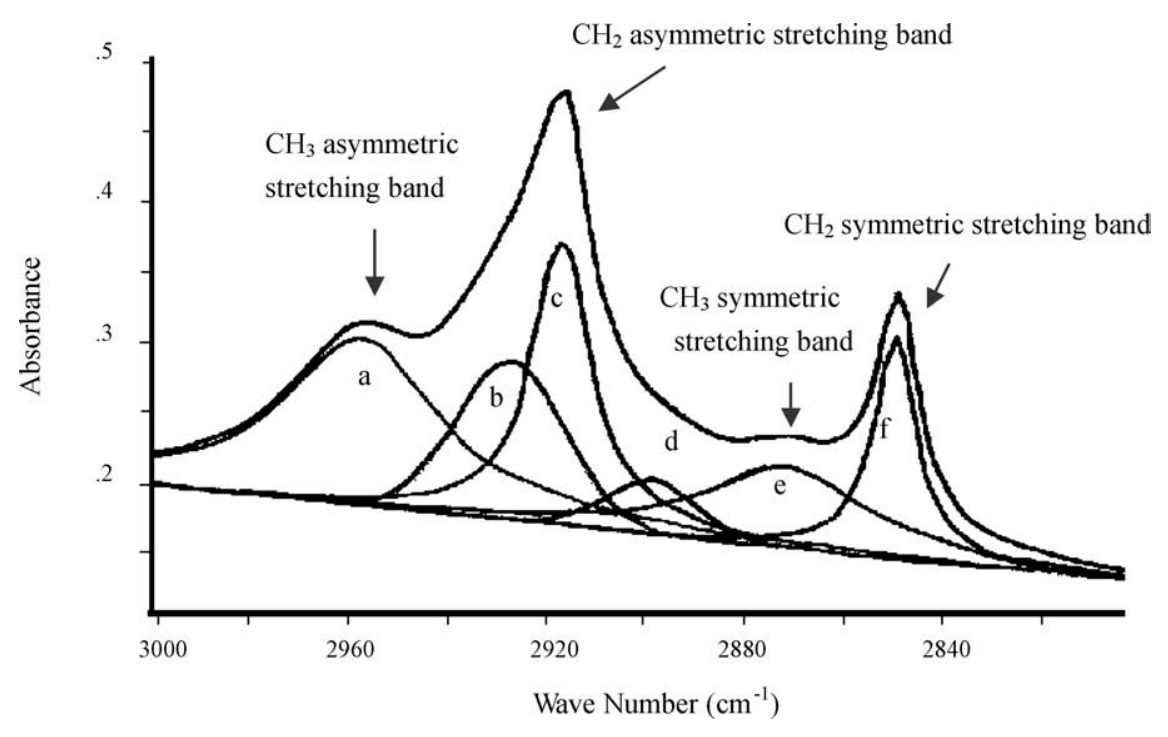

Fig. 2. Spectral analysis by curve-fitting using GRAMS/32 software.

area of the bands in the spectral region of $3000-2800 \mathrm{~cm}^{-1}$ due to the carbon-hydrogen stretching vibrations were analyzed; these were generally the strongest bands in the spectra of lipids with the $\mathrm{CH}_{2} / \mathrm{CH}_{3}$ asymmetric and symmetric stretching modes. Initial parameters for curve-fitting were obtained from fitting a few sample spectra with autofind function and a Gaussian shape was assumed for each absorption band. After each fitting cycle, the bands that contributed to less than $1 \%$ to the overall area under the IR spectrum were cancelled and a new fitting cycle with the remaining free parameters was started. Other fitting functions, such as Lorentzian or Gaussian-Lorentzian were also substituted for each component one by one to start a new fitting cycle. The fitting cycle was completed when the solution reached a minimum. Goodness of fit was determined based on the criteria including reduced $\mathrm{Chi}^{2}$, correlation $\left(R^{2}\right)$ and standard error. Typically, best-fit parameters were obtained with six absorption bands ( $a$ to f) when a Gaussian shape was assumed for components b and d, while Lorentzian for the others. Components a and $\mathrm{f}$ were assigned to $\mathrm{CH}_{3}$ asymmetric stretching and $\mathrm{CH}_{2}$ symmetric stretching vibrations, respectively, while sum of components $\mathrm{b}$, c, and $\mathrm{d}$ was assigned to $\mathrm{CH}_{2}$ asymmetric stretching vibrations (Fig. 2). The same set of initial parameters was applied to all the sample spectra to obtain their own best fit. Spectral characteristics derived for the 4 spectra obtained from a single SC sheet were averages for data presentation and analysis in the following.

\section{Results and discussion}

Figure 3 shows the IR spectra in the range of 2800 to $3000 \mathrm{~cm}^{-1}$ of a single piece of isolated porcine SC sheet progressively lipid extracted with chloroform/methanol mixtures of different volume ratios ( $1: 20$ to $2: 1$ ) for various length of time (10 min to overnight). As expected, the peak absorbance of $\mathrm{CH}_{2}$ symmetric $\left(2850 \mathrm{~cm}^{-1}\right)$ and asymmetric $\left(2920 \mathrm{~cm}^{-1}\right)$ stretching bands decreased gradually upon lipid extraction by solvent treatment. In the first attempt we have used the baseline correction function in the OMNIC software to obtain the integrated peak areas of those bands, as illustrated in Fig. 1. When the peak area of both $\mathrm{CH}_{2}$ symmetric and asymmetric stretching bands obtained as such was 


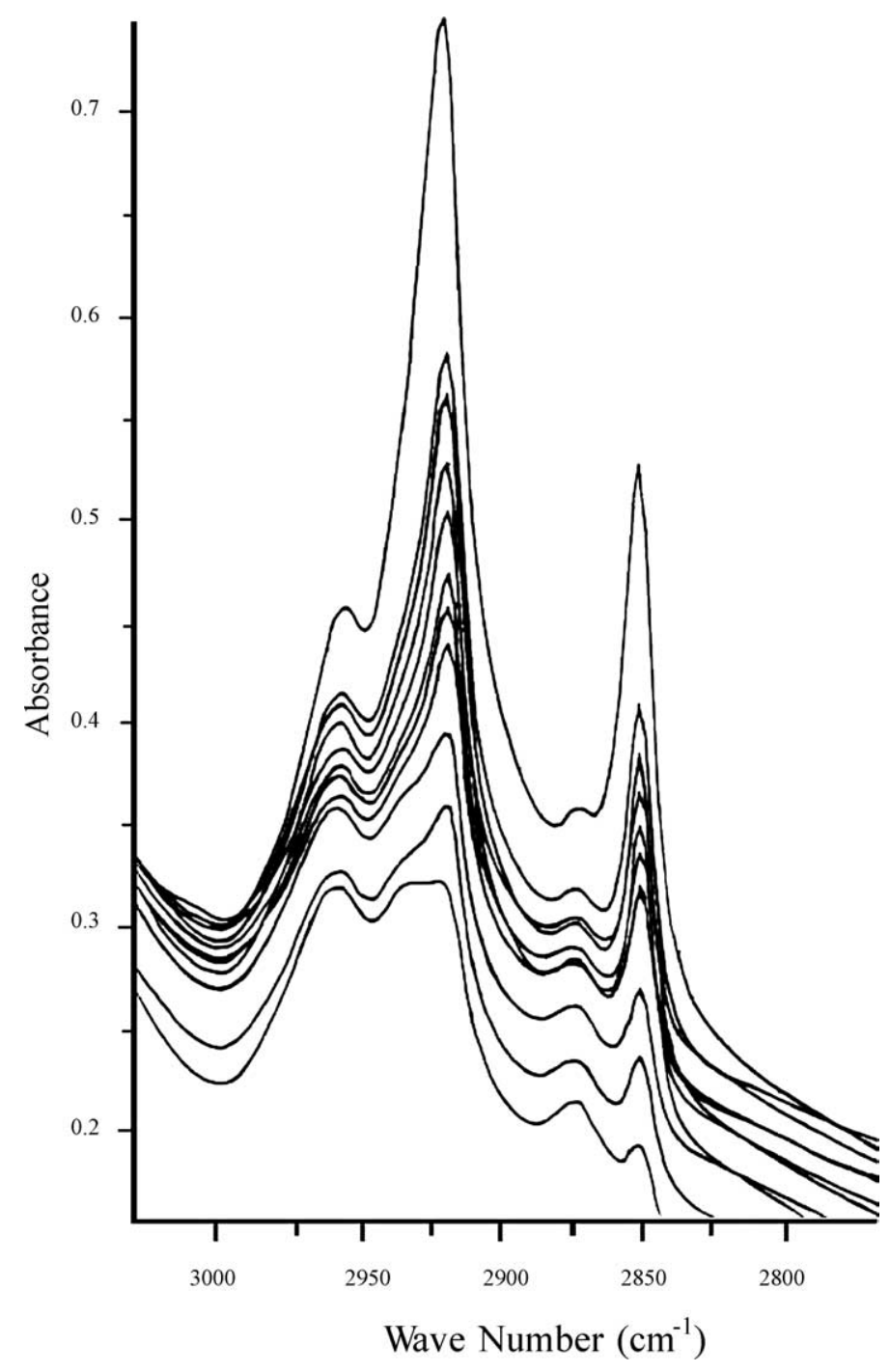

Fig. 3. (From top to bottom) Initial IR spectrum of a single porcine SC sheet and spectra obtained following progressive extractions with chloroform/methanol mixtures of different volume ratios (1:20 to $2: 1)$ for various length of time (10 min to overnight).

plotted as a function of the cumulative amount of lipid removed by solvent extraction, linear relationships were observed $\left(R^{2}=0.91\right.$ and 0.88 , respectively, Fig. 4). The results suggested that the IR spectral features in the region of $2800-3000 \mathrm{~cm}^{-1}$ due to $\mathrm{C}-\mathrm{H}$ stretching vibrations can be used for quantitative measurement of SC lipids weight.

In order to evaluate the quantitative relationship between the IR spectral characteristics and the SC lipid content, IR spectra of 46 pieces of SC sheets obtained from different body sites of the pig were collected. Due to the complexity of those absorption bands originating from SC lipids, curve-fitting procedures were employed to deconvolute the individual bands in the spectral range of 2800 to $3000 \mathrm{~cm}^{-1}$ (Fig. 2). Since preliminary analysis using baseline correction function in the OMNIC software for the spectra obtained in this set of experiments has shown that $\mathrm{CH}_{2}$ asymmetric stretching band yielded bet- 

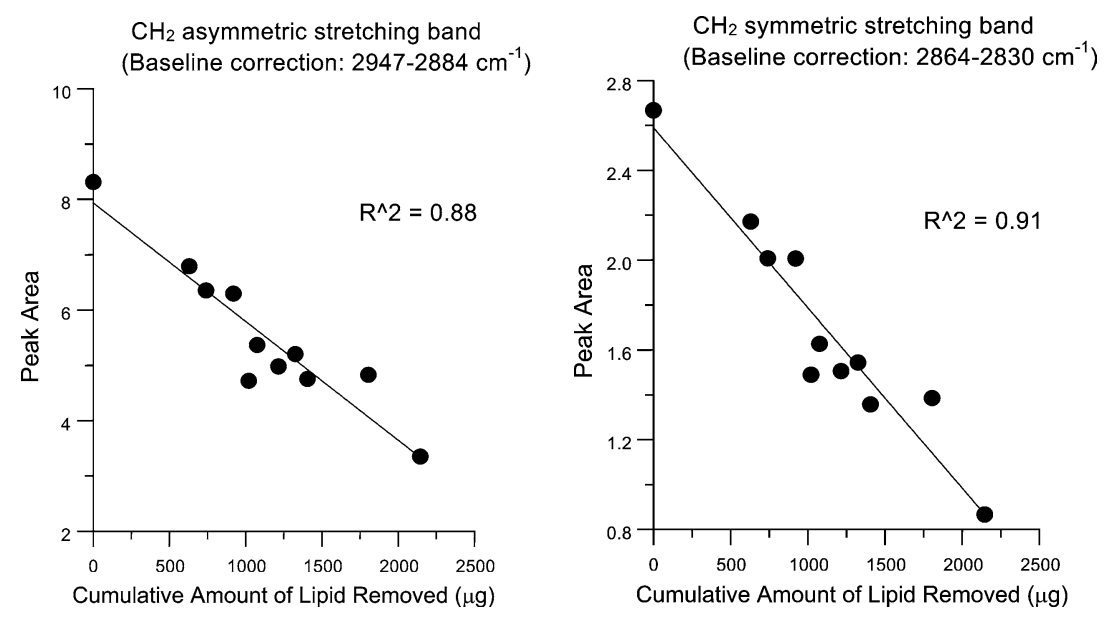

Fig. 4. Peak areas of $\mathrm{CH}_{2}$ symmetric and asymmetric stretching bands in the IR spectra of a single porcine SC sheet progressively extracted with chloroform/methanol mixtures as a function of cumulative amount of lipid removed, with spectra analyzed by baseline correction using OMNIC software.
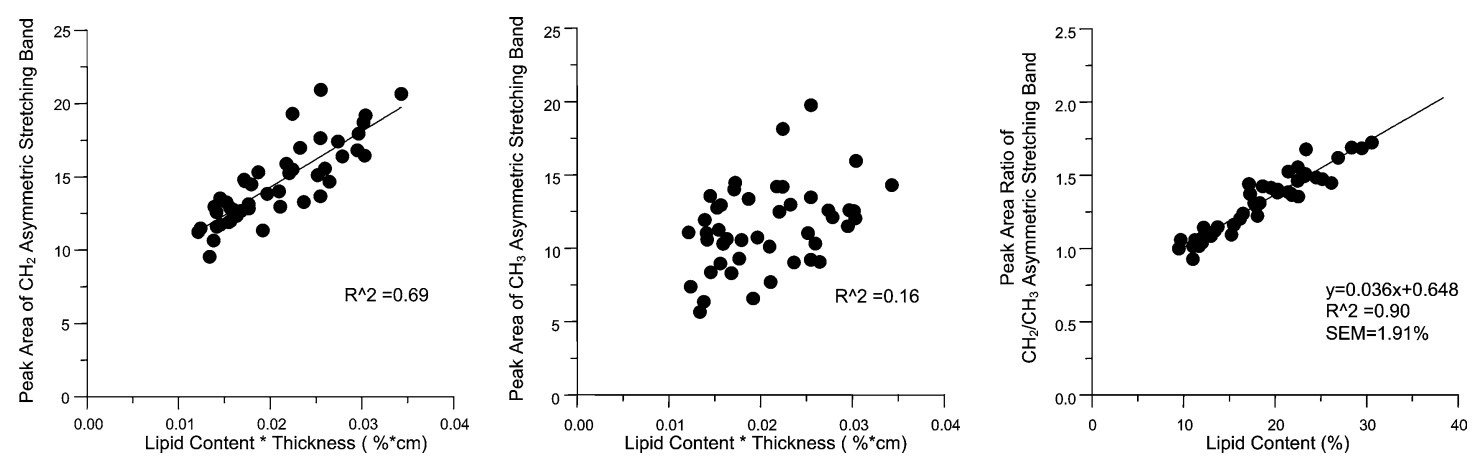

Fig. 5. Peak areas of $\mathrm{CH}_{2}$ and $\mathrm{CH}_{3}$ asymmetric stretching bands in the IR spectra of isolated porcine SC sheets $(n=46)$ as a function of SC lipid content multiplied by thickness, and their ratio as a function of SC lipid content, with spectra analyzed by curve-fitting using GRAMS/32 software.

ter correlation with lipid amount than $\mathrm{CH}_{2}$ symmetric stretching band, the former was selected for the following analysis. Based on Beer-Lambert's Law, the peak areas of $\mathrm{CH}_{2}$ and $\mathrm{CH}_{3}$ asymmetric stretching bands were plotted as a function of SC lipid content multiplied by its thickness (Fig. 5), where SC thickness was determined as SC weight divided by its surface area assuming the SC density of $1 \mathrm{~g} / \mathrm{cm}^{3}$. Results demonstrated that correlation existed with the peak area of $\mathrm{CH}_{2}$ stretching band $\left(R^{2}=0.69\right)$, but to a much less extent with the peak area of $\mathrm{CH}_{3}$ stretching band $\left(R^{2}=0.16\right)$. However, when the peak area ratio of the $\mathrm{CH}_{2} / \mathrm{CH}_{3}$ asymmetric stretching bands was plotted as a function of SC lipid content, a strong linear relationship was observed $\left(y=0.036 x+0.648, R^{2}=0.90\right.$, where $x$ is SC lipid content, and $y$ is the peak area ratio of the $\mathrm{CH}_{2} / \mathrm{CH}_{3}$ asymmetric stretching band). The 46 calculated residues based on the equation were between $-3.93 \%$ and $5.26 \%$. The standard error of measurement calculated as $\sqrt{\sum\left(L_{c}-L_{m}\right)^{2} /(n-1)}$ was $1.91 \%$, where $L_{c}$ is the SC lipid content calculated from the regression line, $L_{m}$ is the gravimetrically measured lipid content, and $n$ is the sample size $(=46)$. It also appeared that the peak areas of $\mathrm{CH}_{3}$ asymmetric stretching band were strongly correlated to $\mathrm{SC}$ thickness linearly $\left(R^{2}=0.92\right.$, Fig. 6). 


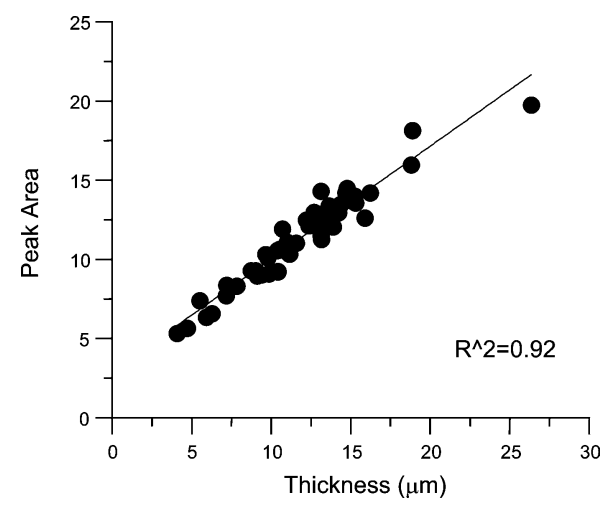

Fig. 6. Peak area of $\mathrm{CH}_{3}$ asymmetric stretching band in the IR spectra of isolated porcine SC sheets as a function of SC thickness, with spectra analyzed by curve-fitting using GRAMS/32 software.

The method described here is simple and highly reproducible as commonly seen with the in vitro FTIR techniques. It is not surprising that the curve-fitting procedures have resolved the $\mathrm{CH}_{2}$ asymmetric stretching band into three components (i.e., b, c, and d in Fig. 2). It has been known in lipid bilayer membranes that, although $\mathrm{CH}_{2}$ asymmetric vibrational mode is a major constituent of IR absorption near $2920 \mathrm{~cm}^{-1}$, the $2920 \mathrm{~cm}^{-1}$ absorption band contains significant overlapping contributions from methyl groups, and the $\mathrm{CH}_{2}$ asymmetric vibrational mode can itself be perturbed by interaction with the first overtones of the methylene scissoring vibration [11]. In contrast, the absorption band near $2850 \mathrm{~cm}^{-1}$ (component f, Fig. 2) is considerably freer of such effects and under most circumstances arises predominantly from the symmetric stretching vibrations of methylene groups. Moreover, because the $\mathrm{CH}_{3}$ asymmetric stretching band was sufficiently well resolved from the $\mathrm{CH}_{2}$ stretching band, and appeared to be directly proportional to $\mathrm{SC}$ thickness, the $\mathrm{CH}_{3}$ asymmetric stretching band could be used as an internal standard against which the peak areas of the neighboring $\mathrm{CH}_{2}$ stretching band was scaled, thereby facilitating comparison and analysis of spectra acquired in $\mathrm{SC}$ with widely varying thickness. It is known that proteins constitute the majority of the dry weight of the SC, and in this study, they represent approximately 70 to $90 \%$ of the total SC weights. Figure 3 also shows that after complete lipid extraction, the $\mathrm{CH}_{3}$ absorption bands, both symmetric and asymmetric, remain relatively constant. We speculate that those two bands originate mainly from protein structure in the SC. The procedures will provide an additional advantage for lipid analysis, especially when used in combination with a fiber-optic accessory in vivo for human SC lipid quantitation, where the thickness of SC spectra collected remained unknown.

The study also showed that both $\mathrm{CH}_{2}$ symmetric and asymmetric stretching frequencies in the IR spectra of isolated porcine SC sheets decreased with increasing SC lipid content (Fig. 7). It has been demonstrated that magnitude of the increase in $\mathrm{CH}_{2}$ stretching frequency correlates with the number of acyl gauche conformers for a number of lipid systems [20], and a remarkable correlation exists between water permeability and the frequency of $\mathrm{CH}_{2}$ stretching vibrations [12]. Our findings further indicate that there is fair correlation existing between conformation order in the hydrocarbon chain of SC lipids and SC lipid content, which is in good agreement with the inverse relationship between water permeability and SC lipid content demonstrated by Elias et al. [21].

In conclusion, the study demonstrated the feasibility of using FTIR technique to rapidly and accurately measure SC lipid content. This FTIR method can be potentially developed as a noninvasive technique for human SC lipid quantitation in vivo with the assistance of a fiber-optic accessory. 

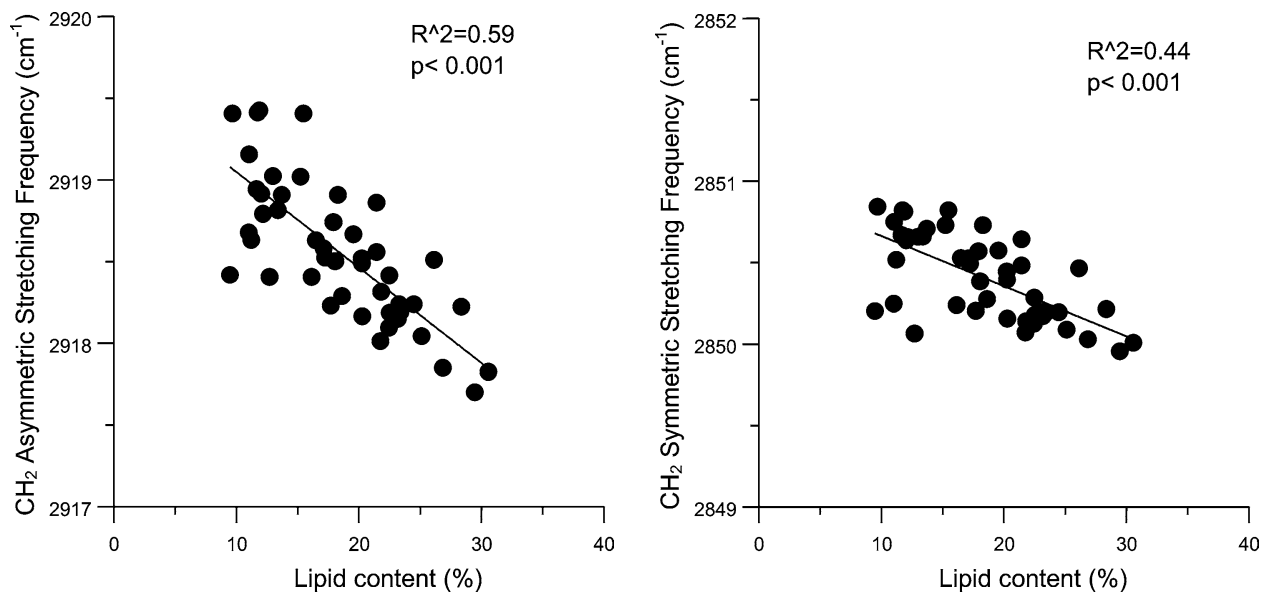

Fig. 7. $\mathrm{CH}_{2}$ symmetric and asymmetric stretching frequencies in the IR spectra of isolated porcine SC sheets $(n=46)$ as a function of SC lipid content.

\section{Acknowledgement}

This work was supported by grant NSC 89-2320-B006-145 from the National Science Council of Taiwan.

\section{References}

[1] R.J. Scheuplein and J.H. Blank, Permeability of the skin, Physiol. Rev. 51 (1971), 702-747.

[2] B. Idson, Percutaneous absorption, J. Pharmaceut. Sci. 64 (1975), 901-924.

[3] P.M. Elias, Epidermal lipids, barrier function, and desquamation, J. Invest. Dermatol. 80 (1983), 44-49.

[4] S.Y.E. Hou, A.K. Mitra, S.H. White, G.K. Menon, R. Ghadially and P.M. Elias, Membrane structures in normal and essential fatty acid deficient stratum corneum: Characterization by ruthenium tetroxide staining and X-ray diffraction, J. Invest. Dermatol. 96 (1991), 215-223.

[5] A. Yamamoto, S. Serizawa, M. Ito and Y. Sato, Stratum corneum lipid abnormalities in atopic dermatitis, Arch. Dermatol. Res. 283 (1991), 219-223.

[6] M. Aszterbaum, G.K. Menon, K.R. Feingold and M.L. Williams, Ontogeny of the epidermal barrier to water loss in the rat: Correlation of function with stratum corneum structure and lipid content, Pediatr. Res. 31 (1992), 308-317.

[7] R. Ghadially, M.L. Williams, S.Y. Hou and P.M. Elias, Membrane structural abnormalities in the stratum corneum of the autosomal recessive ichthyoses, J. Invest. Dermatol. 99 (1992), 755-763.

[8] H.M. Sheu, J.Y.Y. Lee, K.W. Kuo and J.C. Tsai, Permeability barrier abnormality of hairless mouse after topical corticosteroid: Characterization by ruthenium tetroxide staining and high-performance thin-layer chromatography, J. Dermatol. 25 (1998), 281-289.

[9] S. Meguro, Y. Arai, K. Masukawa, K. Uie and I. Tokimitsu, Stratum corneum lipid abnormalities in UVB-irradiated skin, Photochem. Photobiol. 69 (1999), 317-321.

[10] P.W. Wertz and D.T. Downing, Free sphingosine in human epidermis, J. Invest. Dermatol. 94 (1990), 159-161.

[11] R.N.A.H. Lewis and R.N. McElhaney, Fourier transform infrared spectroscopy in the study of hydrated lipids and lipid bilayer membranes, in: Infrared Spectroscopy of Biomolecules, H.H. Mantsch and D. Chapman, eds, Wiley-Liss, New York, 1996, pp. 159-202.

[12] R.O. Potts and M.L. Francoeur, Lipid biophysics of water loss through the skin, Proc. Nat. Acad. Sci. USA 87 (1990), 3871-3873.

[13] T. Kai, V.H.W. Mak, R.O. Potts and R.H. Guy, Mechanism of percutaneous penetration enhancement: Effect of n-alkanols on the permeability barrier of hairless skin, Journal Controlled Release 12 (1990), 103-112.

[14] C.Y. Goates and K. Knutson, Enhanced permeation of polar compounds through human epidermis. I. Permeability and membrane structural changes in the presence of short chain alcohols, Biochim. Biophys. Acta 195 (1994), 169-179. 
[15] Y. Takeuchi, H. Yasukawa, Y. Yamaoka, N. Takahashi, C. Tamura, Y. Morimoto, S. Fukushima and R.C. Vasavada, Effects of oleic acid and propylene glycol on rat abdominal stratum corneum: Lipid extraction and appearance of propylene glycol in the dermis measured by Fourier transform infrared/attenuated total reflectance (FT-IR/ATR) spectroscopy, Chem. Pharmaceut. Bull. 41 (1993), 1434-1437.

[16] D. Bommannan, R.O. Potts and R.H. Guy, Examination of stratum corneum barrier function in vivo by infrared spectroscopy, J. Invest. Dermatol. 95 (1990), 403-408.

[17] P. Franck, J.-L. Sallerin, H. Schroeder, M.-A. Gelot and P. Nabet, Rapid determination of fecal fat by Fourier transform infrared analysis (FTIR) with partial least-squares regression and an attenuated total reflectance accessory, Clin. Chem. 42 (1996), 2015-2020.

[18] G. Janatsch and J.D. Druse-Jarres, Multivariate calibration for assays in clinical chemistry using attenuated total reflection infrared spectra of human blood plasma, Analyt. Chem. 61 (1989), 2016-2023.

[19] M.A. Lampe, A.L. Burlingame, J. Whiteney, M.L. Williams, B.E. Brown, E. Roitman and P.M. Elias, Human stratum corneum lipids: characterization and regional variations, J. Lipid Res. 24 (1983), 120-130.

[20] D.G. Cameron, A. Martin, D.J. Moffatt and H.H. Mantsch, Infrared spectroscopic study of the gel to liquid-crystal phase transition in live Acholeplasma laidlawii cells, Biochemistry 24 (1985), 4355-4359.

[21] P.M. Elias, E.R. Cooper, A. Korc and B.E. Brown, Percutaneous transport in relation to stratum corneum structure and lipid composition, J. Invest. Dermatol. 76 (1981), 297-301. 


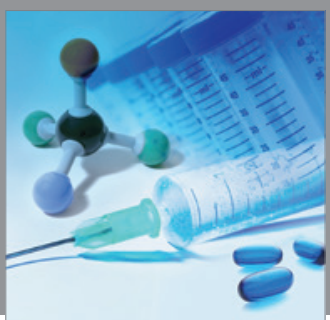

International Journal of

Medicinal Chemistry

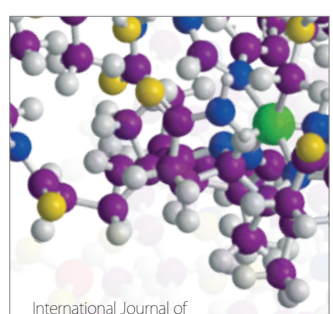

Carbohydrate Chemistry

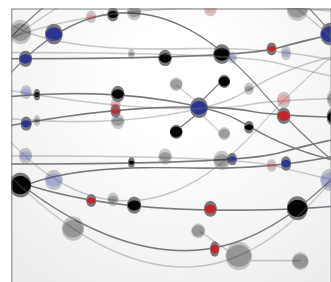

The Scientific World Journal
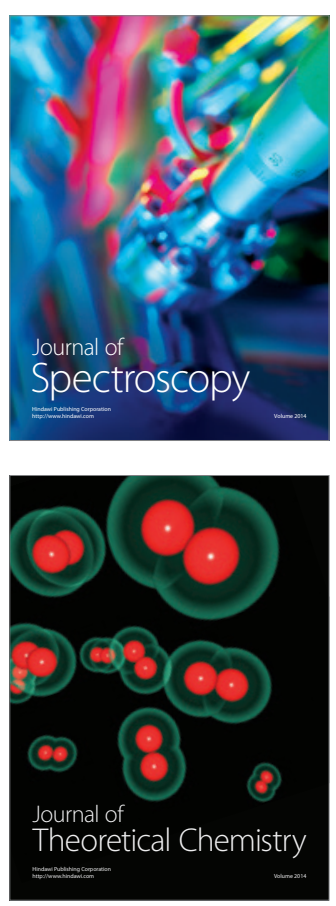
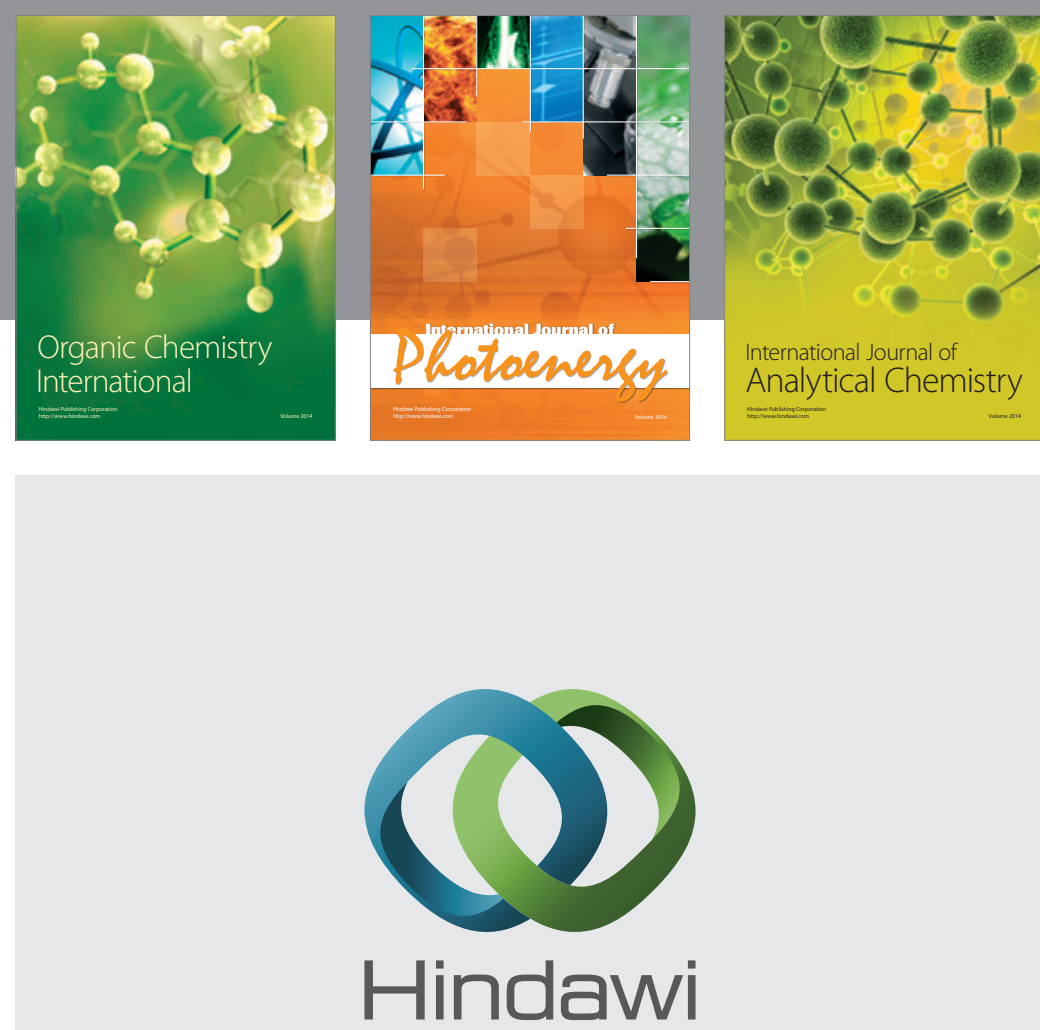

Submit your manuscripts at

http://www.hindawi.com
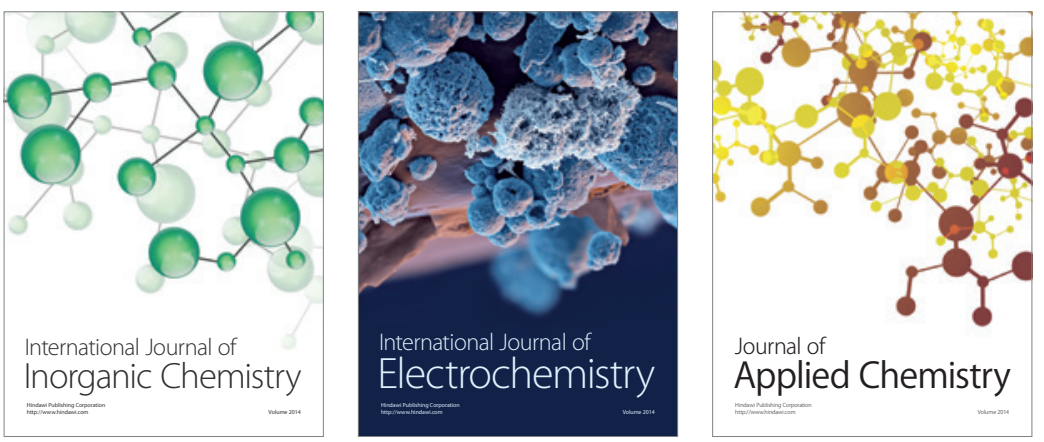

Journal of

Applied Chemistry
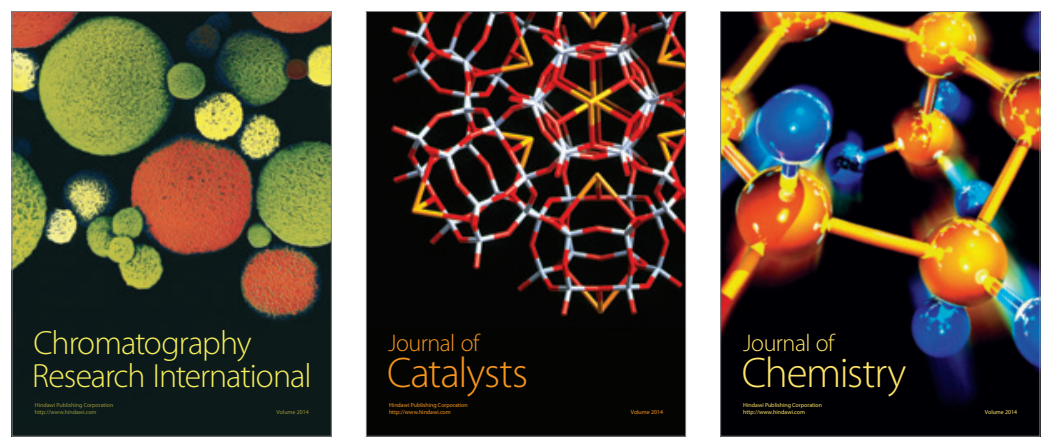
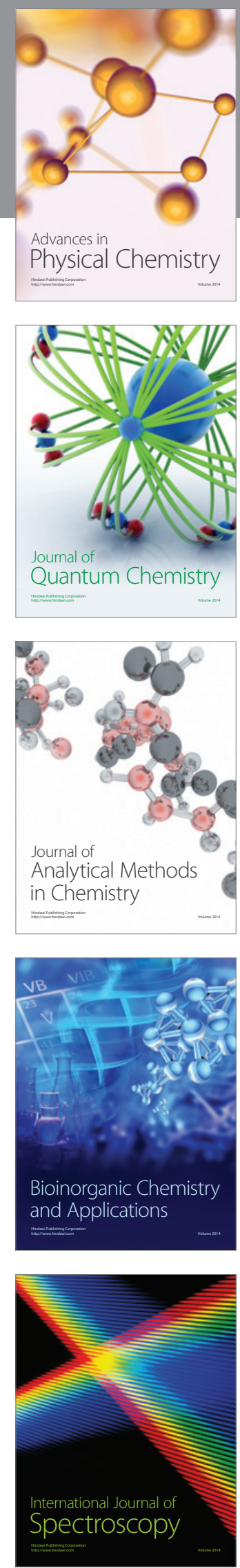